This is a self-archived version of an original article. This version may differ from the original in pagination and typographic details.

Author(s): Samanta, Amit; Chang, Zheng

Title: Adaptive Service Offloading for Revenue Maximization in Mobile Edge Computing with Delay-Constraint

Year: 2019

Version: Accepted version (Final draft)

Copyright: @ 2019 IEEE

Rights: In Copyright

Rights url: http://rightsstatements.org/page//nC/1.0/?language=en

Please cite the original version:

Samanta, A., \& Chang, Z. (2019). Adaptive Service Offloading for Revenue Maximization in Mobile Edge Computing with Delay-Constraint. IEEE Internet of Things Journal, 6(2), 3864-3872. https://doi.org/10.1109/JIOT.2019.2892398 


\title{
Adaptive Service Offloading for Revenue Maximization in Mobile Edge Computing with Delay-Constraint
}

\author{
Amit Samanta, Student Member, IEEE, and Zheng Chang, Senior Member, IEEE
}

\begin{abstract}
Mobile Edge Computing (MEC) is an important and effective platform to offload the computational services of modern mobile applications, and has gained tremendous attention from various research communities. For delay and resource constrained mobile devices, the important issues include: 1) minimization of the service latency; 2) optimal revenue maximization; 3) high quality-of-service $(\mathrm{QoS})$ requirement to offload the computational service offloading. To address the above issues, an adaptive service offloading scheme is designed to provide the maximum revenue and service utilization to MEC. Unlike most of the existing works, we consider both the delay-tolerant and delay-constraint services in order to achieve the optimized service latency and revenue. Furthermore, we consider the different priorities to prioritize the edge services for optimal service offloading. We formulate the proposed scheme mathematically. Simulation results are presented to demonstrate the effectiveness of the proposed adaptive service offloading scheme over other existing state-of-the-art solutions, in terms of service latency, utility value, revenue and utilization.
\end{abstract}

Index Terms-Mobile edge computing, adaptive service offloading, revenue maximization, performance analysis.

\section{INTRODUCTION}

In recent times cloud platform has become very important platform for modern day mobile applications to support Tactile Internet infrastructure [1], [2]. Generally, the conventional standalone applications execute their services fully at the mobile device, whereas cloud applications execute their services at the cloud, as they comprised of multiple components. Hence, one of the components of cloud applications execute at the cloud and another component running on the mobile device, jointly they establish an application available to mobile users [3]. Such mobile cloud applications require high data processing, infrastructures, storage capability that may not be fulfilled on the standalone mobile devices, thus it is necessary to run part of the application in the cloud. Generally, the cloud servers are placed in a centralized data centers. However, the fundamental problem with cloud computing is the higher service latency and intermittent connectivity between the mobile devices and cloud servers, which may not be able to satisfy the real-time services of different emerging applications, such as augmented reality and online traffic monitoring systems. To

A. Samanta is with the Department of Computer Science and Engineering, Indian Institute of Technology Kharagpur, West Bengal - 721302, India. E-mail: amit.samanta049@gmail.com.

Z. Chang is with the Faculty of Information Technology, University of Jyvaskyla, FI-40014 Jyvaskyla, Finland.

E-mail: zheng.chang@jyu.fi. slove this issues, Mobile edge computing (MEC) has become an important and effective platform for different real-time mobile applications [4]-[6]. The fundamental objective of MEC is to design a small-scale cloud platform deployed at the edge of the network, where different mobile edge devices execute their computational services of different applications, like traffic monitoring, healthcare [7]-[14]. Such platforms are placed nearer to the proximity of users to provide seamless and low-latency access to edge services.

In this paper, we study the revenue maximization problem for computational service offloading in MEC platform from a edge service provider's point of view. To execute the computational services, edge devices submit computational offloading requests, containing source and destination addresses and offloading time intervals, to the edge service provider. The edge service provider designs a revenue maximization problem to specify the resource charges to potential edge devices. Edge devices response to the charges by choosing a computational offloading rate to transmit data over the network. This is one of the preliminary work on MEC with the objective of maximizing total revenue, which measures the aggregated service utilization of edge devices. This objective may be in the interest of both edge service providers and edge devices, where the edge service provider and devices want to extract more revenue to maximize the profit level. Thus, our focus here is on designing an adaptive service offloading scheme for revenue maximization. The main contributions of this paper are discussed below.

1. We propose an adaptive service offloading scheme for MEC to maximize the total revenue, while maintaining total utility value of the network. We also present an optimal revenue optimization problem to maximize the profit level of both edge devices and servers for MEC.

2. We consider the delay-sensitive and delay-tolerant edge services in designing the adaptive service offloading algorithm. We also consider the optimal demand of edge devices for efficient service offloading. Thus, we estimate the total demand of edge devices effectively in order to minimize the service latency.

3. Simulation results demonstrate that our algorithm can effectively offload the computational services from edge devices to edge servers. The results also show that the proposed scheme provides higher service utilization while minimizing total service latency. It also yields the best performance, in terms of utility value and total 
revenue, under different performance settings, compared with other solutions.

The rest of the paper is organized as follows. Section II describes the related work. In Section III, we present the system model for MEC. Section IV describes an adaptive service offloading scheme for MEC, in particular, our adaptive service offloading scheme is designed to provide maximum revenue. Section VI conducts extensive simulations to validate our proposed scheme, and Section VII concludes the paper.

\section{Related WORK}

The problem of computational service offloading with optimal resource and delay is a challenging task for MEC. Over the years, only a few researchers have addressed some of the important issues related to this problem. Mao et al. [15] proposed a dynamic computation offloading scheme for MEC with energy-harvesting edge devices. You et al. [16] proposed an energy-efficient resource allocation scheme for service offloading in MEC. Ko et al. [17] proposed a live prefetching scheme for computational service offloading of edge devices. Zhao et al. [18] proposed a task scheduling and resource allocation scheme for delay-bound MEC platform. Zhang et al. [19] an auction-based service provider selection scheme for MEC. Dinh et al. [20] proposed task allocation and frequency scaling for optimal service offloading in MEC. Ti $e t$ al. [21] proposed computational resource allocation scheme for service offloading in edge clouds. Li et al. [22] proposed dataanalysis for IoT applications in MEC to leverage renewable energy. Shekhar et al. [23] proposed a dynamic resource management scheme for mobile edge clouds. Reiter et al. [24] proposed a hybrid edge computing platform to unleash the full potential of MEC platform for IoT applications. Chang et al. [25] proposed energy-efficient optimization framework for computation offloading in fog computing system. Liu et al. [26] proposed a multi-objective optimization problem for computation offloading in fog computing environment. Wu et al. [27] studied the non-orthogonal multiple access-enabled multi-access MEC to minimize the overall-delay of the mobile users, by jointly optimizing the users' offloaded workloads and the NOMA transmission-time. Samanta and Li [28] proposed an optimal economical framework for MEC. Samanta et al. [29] proposed a latency-oblivious distributed task scheduling algorithm for MEC. Samanta and Li [30] proposed a latencyoblivious service offloading scheme for MEC.

In summary, most for the existing studies [15]-[30] mainly focus on the energy-efficient and resource-efficient computational service offloading scheme in MEC. They did not propose any revenue maximization problem for service offloading in MEC to provide the optimal profits to both edge devices and service provider. Thus, for delay- and resource-constraint edge devices, the service offloading scheme is very important in order to provide fair resources and optimal latency to edge devices. This motivates us to design an adaptive service offloading scheme for revenue maximization in MEC platform.

\section{System Model}

Without loss of generality, we assume there are $N$ edge devices denoted by, $\mathcal{E D}=\left\{E D_{1}, E D_{2}, \cdots, E D_{N}\right\}$, existing

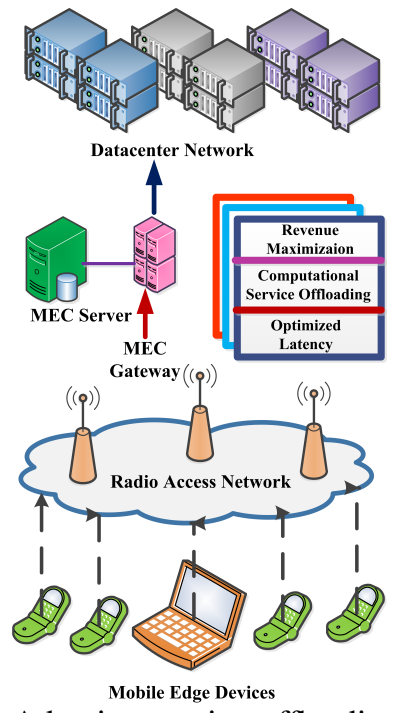

Figure 1: Adaptive service offloading in MEC

in order to offload the computational services, as shown in Figure 1. From the figure, we can see that a set of edge devices are trying to offload their computational services with minimum service delay thorough a backhaul radio access network. Each of the edge device has different kind of services denoted by $\mathbb{S}=\left\{S_{1}, S_{2}, \cdots, S_{K}\right\}$ and they belong to different real-time mobile applications (i.e., self-driving car, augmentedreality, traffic monitoring system etc.). The edge devices offload their computational services to edge servers denoted by, $\mathcal{E S}=\left\{E S_{1}, E S_{2}, \cdots, E S_{M}\right\}$, where each edge server has a certain computational capacity. The owners of the edge servers is considered to be edge service providers denoted by $\mathcal{S P}=\left\{S P_{1}, S P_{2}, \cdots, S P_{O}\right\}$, hence they can ask for prices to execute their services. In this scheme, the computational services $S_{\text {arr }}$ arrive at edge servers by Uniform distribution and the offload of computational services organized according to their priorities. However, the edge devices require fair amount of bandwidths to offload their computational services effectively. We assume that the edge device $E D_{i}$ has a maximum $\mathbb{B}_{i}^{m x}$ and a minimum $\mathbb{B}_{i}^{m y}$ bandwidth requirements to offload the computational services to edge servers.

Fundamentally, the edge devices has very limited power to offload their computational services, therefore the service offloading mechanism is very important to minimize the energy consumption rate of edge devices. Here, we consider the initial energy of a edge device $E D_{i}$ is $\mathcal{E}_{i n i}^{i}$. Along with energy consumption, it is necessary to minimize the service offloading price in order to maximize the revenue of edge service providers and devices. Here, the total service offloading price for a edge device $E D_{i}$ is denoted by $\mathbb{P}_{o f f}^{t}$. Therefore, we propose an adaptive service offloading scheme for MEC to minimize the service latency and offloading price. Thereafter, we propose an adaptive service offloading algorithm to maximize the revenue of edge devices.

\section{Adaptive Service OfFloading}

Due to heavy network load and congestion, the service offloading latency and price increases in the network, which inherently minimizes the service utilization in MEC. In order 
to improve the service utilization and maximize the revenue of edge devices, here we discuss an adaptive service offloading scheme for MEC. At first, we need to estimate the total service latency encountered by edge devices in the network, while offloading the computational services. Later, we propose a utility maximization problem, while taking into consideration of service priority of edge devices.

Approximation of Service Latency. The total service latency encountered by mobile edge devices is estimated based on the total service execution and service offloading latency. They are explained in details below.

- Service Execution Latency: The service execution latency $\mathbb{D}_{E L}^{t}$ of edge device $E D_{i}$ is depended on the total number of CPU cycles required to execute service $S_{i}$ and the local service computing capacity distributed to service $S_{i}$ by edge server. We have,

$$
\mathbb{D}_{E L}^{t}=\left[\frac{\mathcal{G}_{i}}{F_{i}^{l o}}+\mathbb{W}_{i}^{t}\right]
$$

where $\mathcal{G}_{i}$ denotes the total number of CPU cycles required to execute service $S_{i}, F_{i}^{l o}$ denotes local service computing capacity distributed to service $S_{i}$ by edge server and $\mathbb{W}_{i}^{t}$ denotes the initial waiting time to get the required number of CPU cycles required to execute service.

- Service Offloading Latency: The service offloading latency $\mathbb{D}_{o f f}^{t}$ is directly proportional to the total waiting time to offload the computational services on edge devices. It is defined as:

$$
\mathbb{D}_{o f f}^{t}=\frac{\mathbb{J}_{i}^{t}}{q_{i}^{t}}
$$

where $\mathbb{J}_{i}^{t}$ and $q_{i}^{t}$ denote the total service length and time to execute the service $S_{i}$ at time $t$, respectively.

Hence, the total estimated service latency $\mathbb{D}_{t o}^{t}$ for edge device $E D_{i}$ is the addition of both service execution latency $\mathbb{D}_{E L}^{t}$ and service offloading latency $\mathbb{D}_{o f f}^{t}$, which is defined as:

$$
\begin{aligned}
\mathbb{D}_{t o}^{t} & =\mathbb{D}_{E L}^{t}+\mathbb{D}_{o f f}^{t} \\
& =\left[\frac{\mathcal{G}_{i}}{F_{i}^{l o}}+\mathbb{W}_{i}^{t}\right]+\frac{\mathbb{J}_{i}^{t}}{q_{i}^{t}}
\end{aligned}
$$

\section{A. Optimal Revenue Maximization}

After estimation of total service latency, we now model an adaptive service offloading scheme for edge devices to maximize the revenue and service utilization. Suppose, we assume that the computational services require $\mathbb{T}$ slots to offload them efficiently to edge servers. Here, we consider a time frame with different time slots. We describe the length of time slot $T$ and index of time-slot by $t$, where $t \in \mathbb{T}=\{1,2, \cdots$,$\} . In a time-$ slot, if more than one edge device choose a particular channel to offloading their services, then we have used the carrier sense multiple access (CSMA) mechanism to overcome the possible collisions in the network. To maximize the revenue of devices, it is necessary to estimate the total demand and price for computational service offloading.

Definition 1. The demand profile $\mathcal{Z}$ of edge devices is denoted by a set of demand variables, $\mathcal{Z}=\left\{\mathbb{Z}_{1}, \mathbb{Z}_{2}, \cdots, \mathbb{Z}_{\mathbb{N}}\right\}$. The demand variable of a edge device is dependent on the total bandwidth requirement and service execution time. We have,

$$
\mathcal{Z}_{i}=\left\{\mathbb{B}_{i}^{r e q}, t_{i}\right\}
$$

where $\mathbb{B}_{i}^{r e q}$ and $t_{i}$ denote the bandwidth and execution time required to offload the computational services.

Definition 2. The average bandwidth requirement of edge devices is estimated using a classical exponential moving average technique. It is defined as:

$$
\mathbb{B}_{i}^{r e q}=\bar{\lambda}_{i}(t)=\alpha \bar{\lambda}_{i}(t-1)+(1-\alpha) \lambda_{i}(t)
$$

where $\bar{\lambda}_{i}(t)$ denotes the average bandwidth requirement(i.e., average reward) at time $t, \alpha$ denotes the exponential moving average rate, and $\bar{\lambda}_{i}(t-1)$ and $\lambda_{i}(t)$ denote the average bandwidth requirement at time $t-1$ and absolute bandwidth requirement at time $t$.

Definition 3. The service offloading rate of edge device $E D_{i}$ is dependent on the data size of computational services and total number of time-slots required for offloading, which is mathematically defined as:

$$
\mathcal{H}_{i}(\mathcal{Y}, t)=\mathbb{V}_{i} \zeta_{i}(\mathcal{Y}, t)
$$

where $\mathbb{V}_{i}$ denotes the data size of computational services and $\zeta_{i}(\mathcal{Y}, t)$ indicates whether edge device $E D_{i}$ successfully gets a time-slot $t$ with probability $\mathcal{Y}$, which is defined as:

$\zeta_{i}(\mathcal{Y}, t)= \begin{cases}1, & \text { edge device } E D_{i} \text { successfully gets a time-slot }, \\ 0, & \text { otherwise, }\end{cases}$

Definition 4. The probability $\mathcal{Y}$ of getting a time-slot $t$ to offload the computational services is defined as:

$$
\mathcal{Y}= \begin{cases}\prod\left(1-\mathbb{T}_{S_{i}}\right), & \text { if } \mathbb{T}_{S_{i}}>\mathbb{T}_{t h}, \\ 1-\prod\left(1-\mathbb{T}_{S_{i}}\right), & \text { otherwise }\end{cases}
$$

where $\mathbb{T}_{S_{i}}$ and $\mathbb{T}_{\text {th }}$ denote the deadline of service $S_{i}$ and threshold deadline, respectively.

Definition 5. The data size of a computational service for edge device $E D_{i}$ is dependent on the service offloading rate and offloading time. Mathematically,

$$
\mathcal{X}_{i}=\mathcal{H}_{i}(\mathcal{Y}, t) t_{i}^{o f f}
$$

where $t_{i}^{\text {off }}$ denotes the offloading time and $\mathcal{H}_{i}(\mathcal{Y}, t)$ denotes the service offloading rate.

Definition 6. The QoS-level is defined as the ratio of total number of computational services offloaded successfully to edge servers and the total service latency of edge devices. It is defined as:

$$
\mathbb{Q}_{i}=\frac{\mathcal{X}_{i}}{\sum_{i \in N} \sum_{t \in \mathbb{T}} \mathbb{D}_{t o}^{t}}
$$

where $\mathcal{X}_{i}$ denotes data size of a computational service for edge device $E D_{i}$ and $\mathbb{D}_{\text {to }}^{t}$ denotes the total estimated service latency for edge device $E D_{i}$.

Definition 7. The edge service provider charge a price for computational service offloading, which is dependent on the 
inflow and outflow services. Mathematically,

$$
\mathcal{P}_{i}^{o f f}=\sum_{i \in N} \sum_{j \in M} \sum_{t \in \mathbb{T}}\left(\gamma \mathcal{A}_{i j}^{i n}(t) x_{i f}^{t}+\psi \mathcal{B}_{j i}^{\text {out }}(t) y_{o f}^{t}\right),
$$

where $\gamma$ and $\psi$ denote the unit price for both in- and outflow services. $\mathcal{A}_{i j}^{\text {in }}(t)$ and $\mathcal{B}_{j i}^{\text {out }}(t)$ denote the in- and out-flow service offloading at time t. $x_{i f}^{t}$ and $y_{\text {of }}^{t}$ denote the unit service offloading price for up- and down-link at time $t$, respectively.

Definition 8. The edge devices contentiously request for bandwidths to execute their computational services. In order to provide the fair bandwidths, the services are mapped to different edge servers in a datacenter. To map the edge servers, the edge service provider charge a price to execute the services with limited delay. In addition to mapping cost, the edge servers charge other prices for edge servers management. The server management price, $\mathcal{P}_{i}^{s m}$, is dependent on the mapping price $\mathcal{P}_{i}^{\text {map }}$, initial sever development price $\mathcal{P}_{i}^{s d}$, and operational price $\mathcal{P}_{i}^{o p}$, which is defined as:

$$
\mathcal{P}_{i}^{s m}=\mathcal{P}_{i}^{\text {map }}+\mathcal{P}_{i}^{s d}+\mathcal{P}_{i}^{o p},
$$

The price changes to edge devices by edge service provider also incorporates the price of virtual machine (VM) creation, management and migration. Thus, the total price of VM configuration and reconfiguration is expressed as:

$$
\mathcal{P}_{i}^{v m}=\mathcal{P}_{i}^{v m_{c} r e}+\mathcal{P}_{i}^{v m_{m} a n g}
$$

where $\mathcal{P}_{i}^{v m_{c} r e}$ and $\mathcal{P}_{i}^{v m_{m} a n g}$ denote the unit VM creation price and management price, respectively.

The total price $\mathcal{P}_{i}^{\text {tot }}$ charge by edge service provider is defined as, $\mathcal{P}_{i}^{\text {tot }}=\mathcal{P}_{i}^{\text {off }}+\mathcal{P}_{i}^{\text {sm }}+\mathcal{P}_{i}^{\text {vm }}$.

\section{OfFloading Decision Framework}

Using the Definitions $2-8$, we formulate net utility $\mathcal{U}_{i}$ for computational service offloading from edge devices to servers, which is expressed as:

$$
\mathbb{U}_{i}=\left(\Delta_{1} \mathcal{H}_{i}(\mathcal{Y}, t) \mathbb{Q}_{i}+\Delta_{2}\left[\frac{\mathbb{B}_{i}^{r e q}}{\mathbb{B}_{i}^{\text {max }}}-\frac{\mathcal{P}_{i}^{\text {tot }}}{\mathcal{P}_{\max }}\right]\right),
$$

where $\Delta_{1}$ and $\Delta_{2}$ denote the scaling factors for service offloading. $\mathcal{P}_{\max }$ is the maximum price set by edge service providers. Having computed the net utility for each edge device, the edge device with the maximum net utility value emerges as the winner and get to offload its services first than the others. Thus, without the loss of generality, we can formulate the optimization problem as:

$$
\begin{array}{cc}
\text { (P1) : } \underset{t>0, \zeta_{i}(\mathcal{Y}, t) \in\{0,1\}}{\operatorname{maximize}} \sum_{i \in N} \mathbb{U}_{i}, \\
& \mathbb{D}_{t o}^{t} \geq \mathbb{D}_{t h}, i \in N, \\
& \mathbb{B}_{i}^{r e q} \geq \mathbb{B}_{i}^{t h}, i \in N, \\
\text { Subject to } \quad & \mathcal{H}_{i}(\mathcal{Y}, t) \geq \mathcal{H}_{t h}, i \in N, \\
& \mathcal{Q}_{i} \geq \mathcal{Q}_{t h}, i \in N, \\
& \mathcal{P}_{i}^{t o t} \geq \mathcal{P}_{t h}, i \in N,
\end{array}
$$

Detail description of this approach is discussed. (14) presents the primary optimization function for service offloading. (15) describes that the actual service latency, $\mathbb{D}_{t o}^{t}$, is to be greater than the threshold service latency, $\mathbb{D}_{t h}$. The bandwidth requirement of edge device, $\mathbb{B}_{i}^{r e q}$, is to be greater than the threshold bandwidth requirement, $\mathbb{B}_{t h}$, as shown in (16). (17) represents that the service offloading rate of edge device, $\mathcal{H}_{i}(\mathcal{Y}, t)$, is to be grater than the threshold offloading rate, $\mathcal{H}_{t h}$. The QoS-level, $\mathcal{Q}_{i}$, is to be greater than the threshold QoS-level, $\mathcal{Q}_{t h}$, as shown in (19). (17) denotes that the total estimated price for edge device, $\mathcal{P}_{i}^{t o t}$, is to be grater than the threshold price, $\mathcal{P}_{t h}$. Solving the optimization problem using Lagrangian Multipliers, we get,

$$
\begin{array}{r}
\Theta_{\mathbb{U}}=\sum_{i=1}^{N} \frac{\Psi_{i}}{\mathbb{U}_{t h}} \Gamma_{i}\left(\mathcal{H}_{i}(\mathcal{Y}, t), \mathbb{Q}_{i}, \mathbb{B}_{i}^{r e q}, \mathcal{P}_{i}^{t o t}\right) \\
-\xi_{1}\left(\sum_{i=1}^{N} \mathbb{B}_{i}^{r e q}-\mathbb{B}_{i}^{t h}\right)-\xi_{2}\left(\sum_{i=1}^{N} \mathcal{H}_{i}(\mathcal{Y}, t)-\mathcal{H}_{t h}\right) \\
-\xi_{3}\left(\sum_{i=1}^{N} \mathcal{Q}_{i}-\mathcal{Q}_{t h}\right)-\xi_{4}\left(\sum_{i=1}^{N} \mathcal{P}_{i}^{t o t}-\mathcal{P}_{t h}\right) .
\end{array}
$$

where $\xi_{1}, \xi_{2}, \xi_{3}$ and $\xi_{4}$ denote the different constraints for Lagrangian Multipliers and $\Psi_{i}$ denotes priority levels of different services in edge devices. Hence, our main objective is to maximize the value of $\mathbb{U}_{i}$ using the Lagrange Multiplier. We have used gradient descent method to solve the problem. We get the Lagrangian Optimization problem is expressed as:

$$
\begin{array}{r}
\mathcal{L}_{\Theta_{\mathbb{U}}}=\sum_{i=1}^{N} \frac{\Psi_{i}}{\mathbb{U}_{t h}} \mathcal{L}_{\mathbb{U}}\left(\mathcal{H}_{i}(\mathcal{Y}, t), \mathbb{Q}_{i}, \mathbb{B}_{i}^{r e q}, \mathcal{P}_{i}^{t o t}\right) \\
-\xi_{1}\left(\sum_{i=1}^{N} \mathbb{B}_{i}^{r e q}-\mathbb{B}_{i}^{t h}\right)-\xi_{2}\left(\sum_{i=1}^{N} \mathcal{H}_{i}(\mathcal{Y}, t)-\mathcal{H}_{t h}\right) \\
-\xi_{3}\left(\sum_{i=1}^{N} \mathcal{Q}_{i}-\mathcal{Q}_{t h}\right)-\xi_{4}\left(\sum_{i=1}^{N} \mathcal{P}_{i}^{t o t}-\mathcal{P}_{t h}\right) .
\end{array}
$$

Hence, we focus on to optimize $\mathcal{L}_{\mathcal{U}}$ using the Lagrange Multiplier. Thus,

$$
\begin{aligned}
\frac{\delta \mathcal{L}_{\Theta_{U}}}{\delta \mathcal{P}_{i}^{t o t}} & =\sum_{i=1}^{N}-\frac{\Psi_{i} \mathcal{L}_{\mathbb{U}}\left(\mathcal{H}_{i}(\mathcal{Y}, t), \mathbb{Q}_{i}, \mathbb{B}_{i}^{\text {req }}, \mathcal{P}_{i}^{\text {tot }}\right)}{\mathcal{P}_{i}^{\text {tot }}{ }^{2}} \\
\frac{\delta \mathcal{L}_{\Theta_{\Psi}}}{\delta \mathcal{H}_{i}(\mathcal{Y}, t)} & =\sum_{i=1}^{N} \frac{\Psi_{i}}{\mathbb{U}_{t h}} \frac{\delta \mathcal{L}_{\mathbb{U}}\left(\mathcal{H}_{i}(\mathcal{Y}, t), \mathbb{Q}_{i}, \mathbb{B}_{i}^{r e q}, \mathcal{P}_{i}^{t o t}\right)}{\delta \mathcal{H}_{i}(\mathcal{Y}, t)} \\
\frac{\delta \mathcal{L}_{\Theta_{\Psi}}}{\delta \mathbb{Q}_{i}} & =\sum_{i=1}^{N} \frac{\Psi_{i}}{\mathbb{U}_{t h}} \frac{\delta \mathcal{L}_{\mathbb{U}}\left(\mathcal{H}_{i}(\mathcal{Y}, t), \mathbb{Q}_{i}, \mathbb{B}_{i}^{r e q}, \mathcal{P}_{i}^{\text {tot }}\right)}{\delta \mathbb{Q}_{i}} \\
\frac{\delta \mathcal{L}_{\Theta_{\mathbb{U}}}}{\delta \mathbb{B}_{i}^{r e q}} & =\sum_{i=1}^{N} \frac{\Psi_{i}}{\mathbb{U}_{t h}} \frac{\delta \mathcal{L}_{\mathbb{U}}\left(\mathcal{H}_{i}(\mathcal{Y}, t), \mathbb{Q}_{i}, \mathbb{B}_{i}^{r e q}, \mathcal{P}_{i}^{\text {tot }}\right)}{\delta \mathbb{B}_{i}^{r e q}}
\end{aligned}
$$

Using these equations, we obtain the minimum value of $x_{\mathbb{U}}$ to get the optimal revenue for edge devices. To analyze the overall performance, our proposed service offloading scheme is named as - ADORE for edge devices. Here, we discuss the algorithm for the adaptive service offloading scheme for mobile edge devices. As shown in Algorithm 1, first, we need to provide three inputs - set of edge devices $(\mathcal{E D})$, set of 
services $\mathbb{S}$ and total time $\mathcal{T}$. In order to provide optimal revenue and price to edge devices, we propose adaptive service offloading scheme to optimize the service latency and price in the network. Initially, we set the waiting time $T_{w a}$ to 0 . Thereafter, for each edge device $E D_{i}$, we conduct the offloading algorithm. When the total time less than the waiting time, i.e., $\mathcal{T}<T_{w a}$, then we create a demand profile $\mathcal{Z}$ and also approximate the total service latency $\mathbb{D}_{t o}^{t}$. Afterward, we estimate average bandwidth requirement $\mathbb{B}_{i}^{r e q}$ and calculate service offloading rate $\mathcal{H}_{i}(\mathcal{Y}, t)$. Also, we estimate

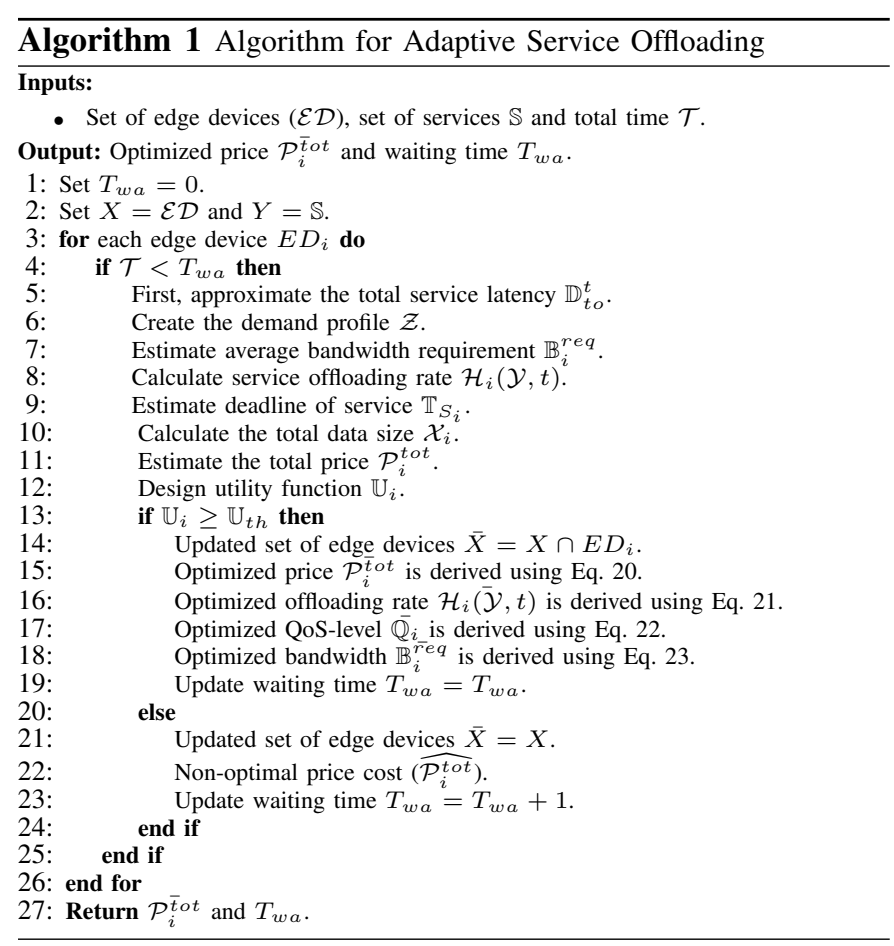

the deadline of a service $\mathbb{T}_{S_{i}}$ and calculate the total data size to be offloaded $\mathcal{X}_{i}$. Further, we estimate the total price $\mathcal{P}_{i}^{\text {tot }}$. Using the estimated and calculated variables, we design a utility function $\mathbb{U}_{i}$ for service offloading. If the utility function $\mathbb{U}_{i}$ greater than the threshold utility function $\mathbb{U}_{t h}$, then we update the set of edge devices $\bar{X}=X \cap E D_{i}$. Also, we update the waiting time $T_{w a}$ is updated as well. Along with, we also get the optimized and optimal price cost $\mathcal{P}_{i}^{\bar{t} o t}$ using Lagrangian Multiplier. Similarly, we can get the optimal values for bandwidth, QoS-level and offloading rate using line 16-18. The process is stopped, when the waiting time crosses a predefined maximum waiting time $T_{w a}^{\max }$. To optimize the revenue price for edge devices using (14), we use the Lagrangian optimization technique to get the optimal value.

Theorem 1. The worst-case computational complexity for ADORE is $O\left(\mathcal{J} N^{2}\right)$, where $N$ is the number of edge devices.

Proof. At first, each edge device tries to offload their computational services to edge servers in order to get the optimal revenues. Therefore, to obtain the optimal revenue, the worst case computation complexity of the service offloading algorithm is $O\left(\mathbb{X} N^{2}\right)$. Before offloading the computational services, the edge devices tries to minimize the offloading latency of network in the absence of multiple edge devices. To minimize the offloading latency, we proposed an service latency approximation algorithm for each edge devices. Hence, the worst case complexity of service latency approximation algorithm is $O(\mathbb{Y} N)$. Thus, combining both the algorithms, we have,

$$
T(n)=\mathbb{L}_{1}\left\{\mathbb{X} T\left(N^{2}\right)+\mathbb{Y} T(N)\right\}+\mathbb{L}_{2} T(1) .
$$

By combining the worst-case complexity for both the algorithms, we obtain, $O\left(\mathcal{J} N^{2}\right)$, where $\mathcal{J}=\mathbb{X}+\mathbb{Y}$. Hence, we observe that the total computational complexity of ADORE in the worst case, is $O\left(\mathcal{J} N^{2}\right)$ with $N$ as the number of edge devices, which completes the proof of Theorem 1 .

\section{Vi. Performance Evaluation}

We present simulation results of the proposed scheme ADORE* in compare to existing schemes. The simulation parameters used in the experiments are shown in Table II.

\section{A. Experimental Setup}

Parameter Settings. we have listed the experiential setup in Table II. We consider 200 edge devices which are distributed over an area of $1000 \mathrm{~m} \mathrm{X} 1000 \mathrm{~m}$ and one macro base station (MBS) co-located to a MEC server. The MEC server located in the MBS, whose computation capability is 100 $\mathrm{GHz}$ and the computation capability of edge device is 0.7 GHz. Each base station has 50 orthogonal wireless channels for the computational service offloading from edge devices to edge servers. Here, the cellular backhaul delay coefficient is considered to be $0.0001 \mathrm{sec} / \mathrm{KB}$ [31]. The total time duration

Table I: Experimental Parameters

\begin{tabular}{l|c}
\hline \multicolumn{1}{c|}{ Parameter } & Value \\
\hline \hline Bandwidth & $20 \mathrm{MHz}$ \\
Total number of CPU cycles of computation task & $1,000 \mathrm{Megacycles}$ \\
Service deadline & {$[4000,6000] \mathrm{ms}$} \\
Computation resource demand & {$[10,20] \mathrm{MHz}$} \\
Transmission power of edge device & $100 \mathrm{mWatts}$ \\
Computation capability of edge device & $0.7 \mathrm{GHz}$ \\
Computation capability of the MEC server & $100 \mathrm{GHz}$ \\
Data traffic arrival modeled as Poisson process & {$[0,10] \mathrm{unit} / \mathrm{sec}$} \\
Expected size of data traffic & $100 \mathrm{Mbits}$ \\
Computation service arrival (mean size $=1 \mathrm{Mbit})$ & {$[0,10]$,} \\
\hline
\end{tabular}

to offload the computation services of mobile edge devices are randomly distributed between 5 and $10 \mathrm{~ms}$. The corresponding computation file size of each computational service varies within the range 300 and $800 \mathrm{~KB}$. The delay requirements of edge devices is considered to be within the range $0.5-1 \mathrm{~s}$.

Table II: Experimental Settings

\begin{tabular}{|c|c|c|c|c|c|c|}
\hline No. & $N$ & $M$ & $\mathcal{P}_{i}^{\text {tot }}$ & $\mathcal{S}_{i}$ & $\Psi_{i}$ & $\left|S_{\text {arr }}\right|$ \\
\hline \hline I & 200 & $10-15$ & {$[1-300]$} & {$[5-10]$} & {$[0,1]$} & {$[0.2-0.6]$} \\
\hline II & 200 & $5-10$ & {$[1-200]$} & {$[10-15]$} & {$[0,1]$} & {$[0.4-0.8]$} \\
\hline III & 200 & $15-20$ & {$[1-400]$} & {$[15-20]$} & {$[0,1]$} & {$[0.5-1.0]$} \\
\hline
\end{tabular}

*The ADaptive service Offloading for Revenue maximization scheme for MEC is called ADORE. 


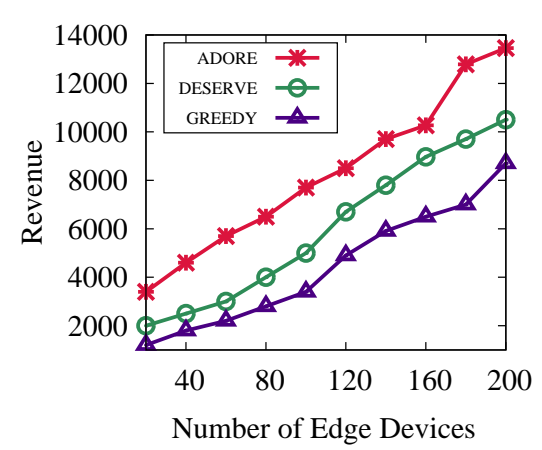

(a) Total Revenue (settings I)

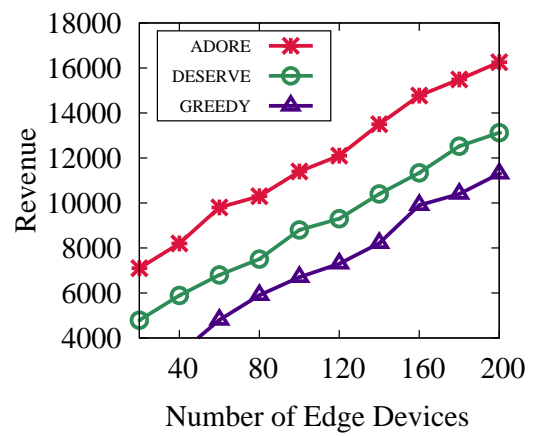

(b) Total Revenue (settings II)

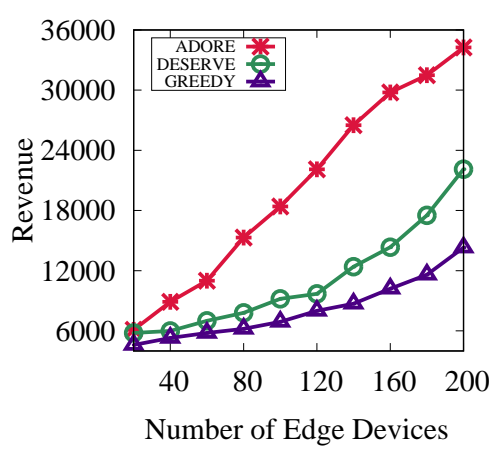

(c) Total Revenue (settings III)

Figure 2: Analysis of total revenue with settings I, II, and III

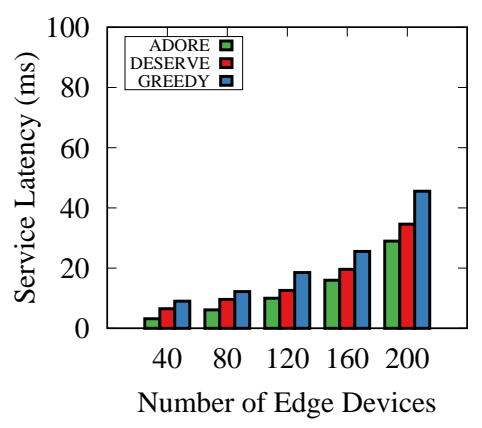

(a) Total Revenue (settings I)

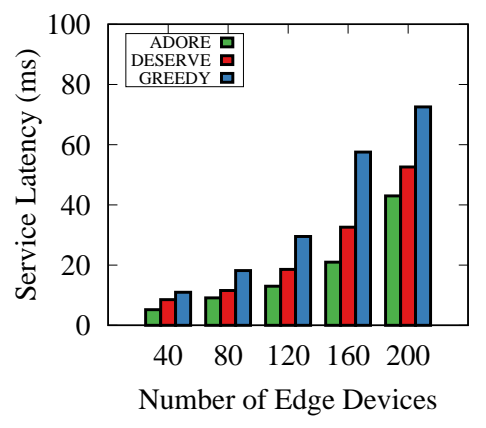

(b) Service Latency (settings I)

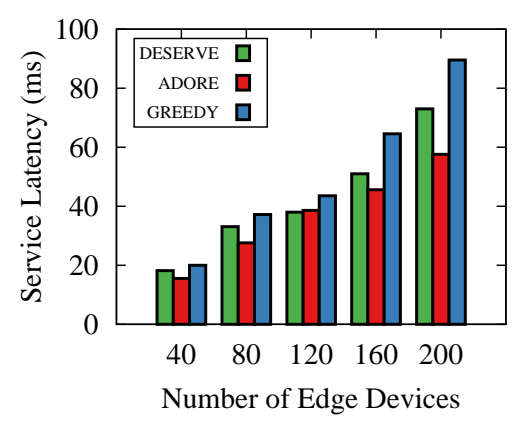

(c) Service Latency (settings III)

Figure 3: Analysis of total service latency with settings I, II, and III

Workload. We implement our scheme in 10 servers, each machine configured with Intel core-i5 processor and $1.7 \mathrm{GHz}$ CPU. For this work, we consider two types of traffic workloads - delay-sensitive (i.e., edge services) and delay-tolerant (i.e., cloud services) workloads. Here, the higher priority is given to delay-sensitive traffic than delay-tolerant traffic workloads. Thus, in our setup, we ran edge services at a higher priority than the normal and background services, respectively.

Metrics. The service latency in this work is defined as the total time required to offload the services from edge devices to edge servers. Finally, we design service utilization metric to measure the efficiency of the proposed scheme. The service utilization is defined as the ratio of the total number of services successfully offloaded and total number of services to be offloaded.

Utilization $=\frac{\text { Total number of services successfully offloaded }}{\text { Total services to be offloaded }}$

Larger values indicate the better performance of ADORE, and if the services face the lesser values then it reduces the service utilization. For computational service offloading, we also measure the revenue of the edge devices, and compare ADORE against with other existing schemes.

Benchmarks. To evaluate the performance, we use two benchmarks - DESERVE [32] and GREEDY. DESERVE [32] proposed a delay-agnostic service offloading scheme for MEC. They implemented a boosting algorithm using software defined networks (SDN), which tries to assign the optimal resources to edge devices and also minimizes the service delay of edge devices. However, they do not consider any resource agnostic property of edge device. We also compared with a GREEDY approach of computational service offloading, here it follows a heuristic solution to find a local optima at each stage with the aim of finding a global optima.

\section{B. Results and Discussion}

Impact on Total Revenue. Here, we compare the revenue of our proposed scheme - ADORE with two offloading baselines. The figures show that ADORE provides better revenue than the existing schemes - DESERVE and GREEDY under both setting I, II, and III. Figure 2(a) shows the revenue of the proposed scheme - ADORE for settings I. From the figure, we observe that revenue increases with the increase in the number of edge devices. As the number of edge devices increases then the offloadable services executed by the each edge devices increases, which inherently increases the revenue. However, our proposed scheme - mISO manages to offload the edge services to edge servers form edge devices efficiently, which eventually increases the total revenue. However, ADORE outperforms the other approaches - DESERVE and GREEDY by $5-9 \%$, respectively. Figure 2(b) shows the social-welfare of the proposed scheme for settings II. We see that the revenue increases with the increase in the number of edge devices. As the number of services increases, the edge devices and servers get to execute more number of services, hence it inherently 


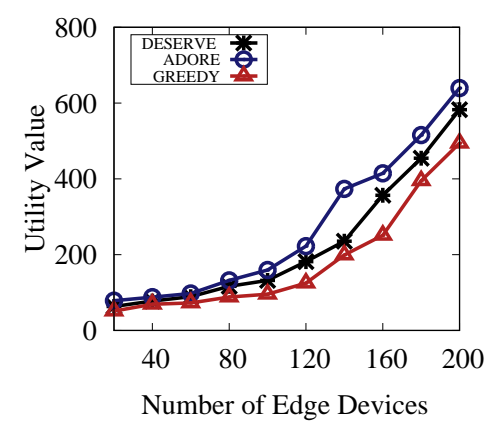

(a) Utility Value (settings I)

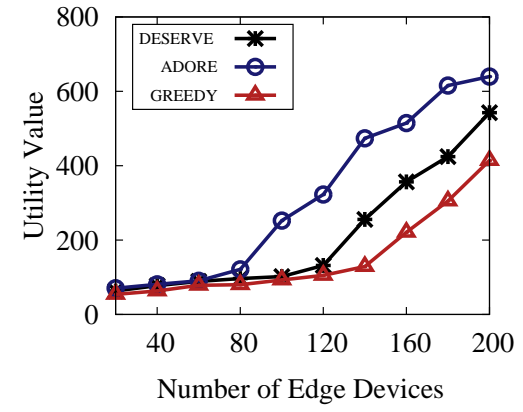

(b) Utility Value (settings II)

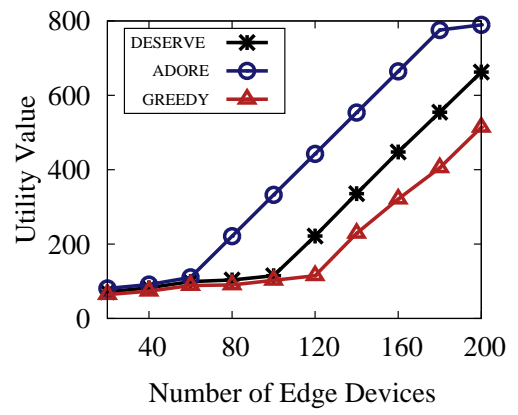

(c) Utility Value (settings III)

Figure 4: Analysis of utility value with settings I, II, and III

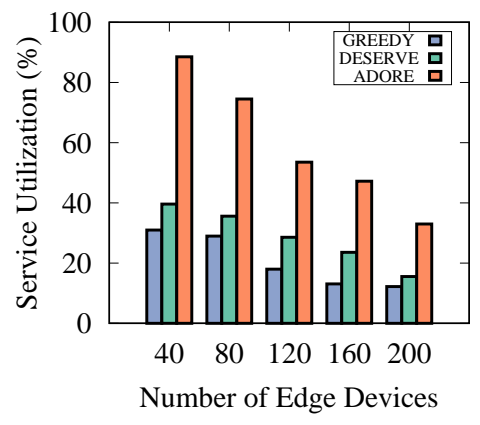

(a) Service Utilization (settings I)

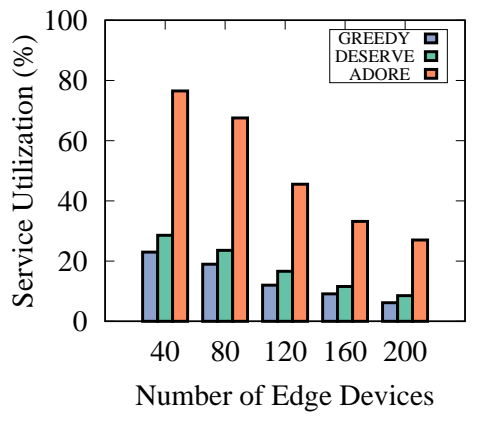

(b) Service Utilization (settings II)

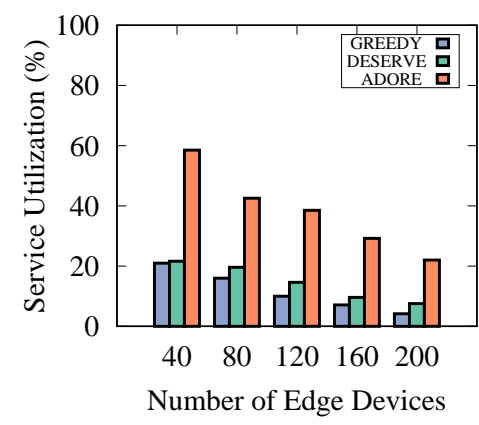

(c) Service Utilization (settings III)

Figure 5: Analysis of service utilization with settings I, II, and III

increases the revenue. However, it is relatively higher than the existing approaches - DESERVE and GREEDY. Hence, the revenue using the proposed scheme - ADORE is higher than other approaches by $18-23 \%$. Figure 2(c) shows the socialwelfare of the proposed scheme for settings III. We see that the revenue increases with the increase in the number of edge devices. As the number of edge servers increases, the edge devices get to execute more number of services in a particular time instant, thus the revenue increases. However, we see that the revenue is higher compared to existing approaches - DESERVE and GREEDY. Hence, the revenue using the proposed scheme - ADORE is higher than other approaches by $21-28 \%$.

Impact on Service Latency. Figure 3(a) shows that the service latency of edge devices, which increases due to increase in real-time IoT applications for settings I. As the edge device increases, therefore the total number of services also increases in the network, which inherently increases the service latency of edge devices. However, the proposed scheme - ADORE outperforms the existing schemes - DESERVE and GREEDY. Hence, the service latency of edge devices using the proposed scheme - ADORE is lesser than other approaches by 4-6\%. Similarly, figure $3(\mathrm{~b})$ shows that the service latency of the network with the increase in the number of edge devices using settings II. As the edge devices increases then the congestion in the network also increases, which increases the service latency. However, ADORE outperforms the other approaches - DESERVE and GREEDY by 6-8 \%, respectively. Similarly, figure 3(c) shows that the service latency of the network with the increase in the number of edge devices using settings III. As the edge services increases, the contention among edge devices also increases to offload their services optimally, which inherently increases the offloading latency. Thus, the total latency increases. However, ADORE outperforms the other approaches - DESERVE and GREEDY by 8-9 \%, respectively.

Impact on Net Utility. Figure 4(a) shows the net utility of the proposed scheme - ADORE for settings I. From the figure, we observe that net utility increases with the increase in the number of edge devices. As the number of edge devices increases the service offloading rate and bandwidth requirement also increases. Hence, the net utility also increases, as it a function of both service offloading rate and bandwidth requirement. Therefore, our proposed scheme - ADORE efficiently manages to offload the service to edge servers using this metric. Hence, ADORE outperforms the other approaches - DESERVE and GREEDY by $15-24 \%$, respectively. Similarly, we tries to find out the offloading decision for settings II in figure 4(b). It also outperforms the existing approaches - DESERVE and GREEDY by $17-19 \%$, respectively. Similarly, we tries to find out the offloading decision for settings III in figure 4(c). ADORE outperforms the existing approaches - DESERVE and GREEDY by $20-23 \%$, respectively.

Impact on Service Utilization. Figure 5(a) shows the service utilization of the proposed scheme using settings I. We see that the service utilization increases with the increase in the number of edge devices. As the number of edge devices increases, then 
the designed platform process and executes more number of services at edge servers. Hence, the utilization of the designed platform using the proposed scheme - ADORE is higher than other approaches by $25-33 \%$. Similarly, figure 5(b) shows that the utilization of the proposed scheme - ADORE using settings II. However, the proposed scheme - ADORE outperforms the existing schemes - DESERVE and GREEDY. Hence, the service utilization of designed platform using the proposed scheme - ADORE is higher than other schemes by 28-38 $\%$. Further, figure 5(c) shows the utilization of the proposed scheme - ADORE using settings III. As the edge devices and services increases in the network, then the edge devices do not get the enough resources to offload their all services, which inherently decreases the service utilization. However, the service utilization of designed platform using the proposed scheme - ADORE is higher than other schemes by $28-38 \%$.

\section{CONCLUSION}

In this work, we proposed an adaptive service offloading scheme for MEC platform in the presence of multiple edge devices. Firstly, we proposed an optimal service offloading scheme to provide a fair amount of resources to edge devices for efficient service offloading. We also propose a utility maximization scheme to minimize the service latency and price for service offloading. The proposed approach shows remarkable development in terms of net utility, service utilization and revenue. As future work, we will implement the proposed approach with real-bed information and hardware implication. We also propose to have an optimal data dissemination scheme for edge devices in the presence of mobility.

\section{REFERENCES}

[1] M. Simsek, A. Aijaz, M. Dohler, J. Sachs, and G. Fettweis, "5g-enabled tactile internet," IEEE Journal on Selected Areas in Communications, vol. 34, no. 3, pp. 460-473, 2016.

[2] A. Aijaz, "Towards 5G-enabled Tactile Internet: Radio resource allocation for haptic communications," in Proc. of IEEE Wireless Communications and Networking Conference Workshops, April 2016, pp. 145-150.

[3] S. Wang, M. Zafer, and K. K. Leung, "Online Placement of MultiComponent Applications in Edge Computing Environments," IEEE Access, vol. 5, pp. 2514-2533, 2017.

[4] P. Garcia Lopez, A. Montresor, D. Epema, A. Datta, T. Higashino, A. Iamnitchi, M. Barcellos, P. Felber, and E. Riviere, "Edge-Centric computing: Vision and challenges," ACM SIGCOMM Computer Communication Review, vol. 45, no. 5, pp. 37-42, 2015.

[5] M. Satyanarayanan, P. Simoens, Y. Xiao, P. Pillai, Z. Chen, K. Ha W. Hu, and B. Amos, "Edge Analytics in the Internet of Things," IEEE Pervasive Computing, vol. 14, no. 2, pp. 24-31, 2015.

[6] A. Ahmed and E. Ahmed, "A Survey on Mobile Edge Computing," in Proc. of International Conference on Intelligent Systems and Control, 2016, pp. 1-8.

[7] A. Samanta, S. Bera, and S. Misra, "Link-Quality-Aware Resource Allocation With Load Balance in Wireless Body Area Networks," IEEE Systems Journal, vol. 12, no. 1, pp. 74-81, 2018.

[8] A. Samanta and S. Misra, "Energy-Efficient and Distributed Network Management Cost Minimization in Opportunistic Wireless Body Area Networks," IEEE Transactions on Mobile Computing, 2017.

[9] S. Misra and A. Samanta, "Traffic-aware efficient mapping of wireless body area networks to health cloud service providers in critical emergency situations," IEEE Transactions on Mobile Computing, pp. 1-1, 2018.

[10] A. Samanta and S. Misra, "Dynamic Connectivity Establishment and Cooperative Scheduling for QoS-Aware Wireless Body Area Networks," IEEE Transactions on Mobile Computing, vol. PP, no. 99, pp. 1-1, 2018.
[11] A. Samanta, Y. Li, and S. Chen, "QoS-aware heuristic scheduling with delay-constraint for WBSNs," in 2018 IEEE International Conference on Communications (ICC). IEEE, 2018, pp. 1-7.

[12] A. Samanta and S. Misra, "Erem: Energy-efficient resource management in body area networks with fault tolerance," in IEEE Global Communications Conference (GLOBECOM). IEEE, 2017, pp. 1-6.

[13] A. Samanta, S. Misra, and M. S. Obaidat, "Wireless body area networks with varying traffic in epidemic medical emergency situation," in IEEE International Conference on Communications (ICC). IEEE, 2015, pp. 6929-6934.

[14] A. Samanta and Y. Li, "Distributed Pricing Policy for Cloud-Assisted Body-to-Body Networks with Optimal QoS and Energy Considerations," IEEE Transactions on Services Computing, 2018.

[15] Y. Mao, J. Zhang, and K. B. Letaief, "Dynamic Computation Offloading for Mobile-Edge Computing with Energy Harvesting Devices," IEEE Journal on Selected Areas in Communications, vol. 34, no. 12, pp. 35903605, 2016.

[16] C. You, K. Huang, H. Chae, and B.-H. Kim, "Energy-efficient Resource Allocation for Mobile-Edge Computation Offloading," IEEE Transactions on Wireless Communications, vol. 16, no. 3, pp. 1397-1411, 2017.

[17] S.-W. Ko, K. Huang, S.-L. Kim, and H. Chae, "Live Prefetching for Mobile Computation Offloading," IEEE Transactions on Wireless Communications, vol. 16, no. 5, pp. 3057-3071, 2017.

[18] T. Zhao, S. Zhou, X. Guo, and Z. Niu, "Tasks scheduling and resource allocation in heterogeneous cloud for delay-bounded mobile edge computing," in Proc. of IEEE International Conference on Communications. IEEE, 2017, pp. 1-7.

[19] H. Zhang, F. Guo, H. Ji, and C. Zhu, "Combinational auction-based service provider selection in mobile edge computing networks," IEEE Access, vol. 5, pp. 13 455-13 464, 2017.

[20] T. Q. Dinh, J. Tang, Q. D. La, and T. Q. Quek, "Offloading in Mobile Edge Computing: Task Allocation and Computational Frequency Scaling," IEEE Transactions on Communications, 2017.

[21] N. T. Ti and L. B. Le, "Computation offloading leveraging computing resources from edge cloud and mobile peers," in IEEE International Conference on Communications. IEEE, 2017, pp. 1-6.

[22] Y. Li, A.-C. Orgerie, I. Rodero, M. Parashar, and J.-M. Menaud, "Leveraging Renewable Energy in Edge Clouds for Data Stream Analysis in IoT," in Proc. of IEEE/ACM International Symposium on Cluster, Cloud and Grid Computing, 2017, pp. 186-195.

[23] S. Shekhar and A. Gokhale, "Dynamic Resource Management Across Cloud-Edge Resources for Performance-Sensitive Applications," in Proc. of IEEE/ACM International Symposium on Cluster, Cloud and Grid Computing, 2017, pp. 707-710.

[24] A. Reiter, B. Prünster, and T. Zefferer, "Hybrid Mobile Edge Computing: Unleashing the Full Potential of Edge Computing in Mobile Device Use Cases," in Proc. of IEEE/ACM International Symposium on Cluster, Cloud and Grid Computing, 2017, pp. 935-944.

[25] Z. Chang, Z. Zhou, T. Ristaniemi, and Z. Niu, "Energy efficient optimization for computation offloading in fog computing system," in Proc. of IEEE Global Communications Conference, Dec 2017, pp. 1-6.

[26] L. Liu, Z. Chang, X. Guo, S. Mao, and T. Ristaniemi, "Multiobjective optimization for computation offloading in fog computing," IEEE Internet of Things Journal, vol. 5, no. 1, pp. 283-294, Feb 2018.

[27] Y. Wu, K. Ni, C. Zhang, L. Qian, and D. H. K. Tsang, "NOMA Assisted Multi-Access Mobile Edge Computing: A Joint Optimization of Computation Offloading and Time Allocation," IEEE Transactions on Vehicular Technology, pp. 1-1, 2018.

[28] A. Samanta and Y. Li, "Time-to-Think: Optimal Economic Considerations in Mobile Edge Computing: Poster," in INFOCOM, 2018.

[29] A. Samanta, Z. Chang, and Z. Han, "Latency-Oblivious Distributed Task Scheduling for Mobile Edge Computing," in IEEE GLOBECOM, 2018 , pp. 1-7.

[30] A. Samanta and Y. Li, "Poster: Latency-Oblivious Incentive Service Offloading in Mobile Edge Computing," in Proc. of ACM/IEEE Symposium on Edge Computing, 2018.

[31] K. Zhang, Y. Mao, S. Leng, Q. Zhao, L. Li, X. Peng, L. Pan, S. Maharjan, and Y. Zhang, "Energy-Efficient Offloading for Mobile Edge Computing in 5G Heterogeneous Networks," IEEE Access, vol. 4, pp. 5896-5907, 2016.

[32] A. Samanta and Y. Li, "DeServE: Delay-agnostic Service Offloading in Mobile Edge Clouds: Poster," in Proc. of ACM/IEEE Symposium on Edge Computing, 2017, pp. 24:1-24:2. 


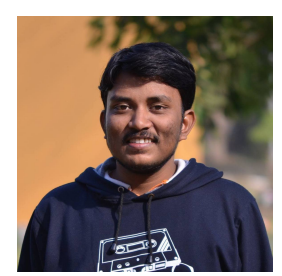

Amit Samanta is presently pursuing his M.S. from the Department of Computer Science and Engineering, Indian Institute of Technology Kharagpur, India. He has worked as a Visiting Research Scholar in Department of Electronic Engineering, Tsinghua University. Besides, he has also worked as a junior project officer in Virtual Lab on Advanced Network Technologies funded by MHRD, Government of India. He received the B.Tech degree in Electronics and Communication Engineering from West Bengal University of Technology, India. His current research interest includes wireless body area networks, data center networks, cloud computing and mobile edge computing. He is a student member of the IEEE.

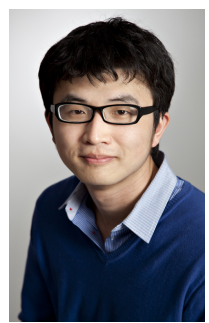

Zheng Changg (S'10-M'13-SM'17) received the B.Eng. degree from Jilin University, Changchun, China in 2007, M.Sc. (Tech.) degree from Helsinki University of Technology (Now Aalto University), Espoo, Finland in 2009 and Ph.D degree from the University of Jyväskylä, Jyväskylä, Finland in 2013. Since 2008, he has held various research positions at Helsinki University of Technology, University of Jyväskylä and Magister Solutions Ltd in Finland. During June to August in 2013, he was a visiting researcher at Tsinghua University, China and during April to May in 2015, he was a visiting researcher at University of Houston, TX. He has been awarded by the Ulla Tuominen Foundation, the Nokia Foundation and the Riitta and Jorma J. Takanen Foundation for his research excellence. He serves as editor of IEEE Access, Springer Wireless Networks and IEEE MMTC Communications Frontier, and guest editor of IEEE Wireless Communications, IEEE Communications Magazine, IEEE Internet of Things Journal, IEEE Networks, EURASIP Journal on Wireless Communications and Networking, Physical Communications and Wireless Communications and Mobile Computing. He also served as TPC member for many IEEE major conferences, such as INFOCOM, ICC and Globecom. He has received Best Paper awards from IEEE Technical Committee on Green Communications \& Computing (TCGCC) and 23rd Asia-Pacific Conference on Communications (APCC) in 2017. He was also named the exemplary reviewer of IEEE Wireless Communications Letter in 2017. Currently he is working as Assistant Professor at University of Jyväskylä and his research interests include IoT, cloud/edge computing, security and privacy, vehicular networks, and green communications. 